\title{
A PRIME IDEAL PRINCIPLE FOR TWO-SIDED IDEALS
}

\author{
MANUEL L. REYES
}

\begin{abstract}
Many classical ring-theoretic results state that an ideal that is maximal with respect to satisfying a special property must be prime. We present a "Prime Ideal Principle" that gives a uniform method of proving such facts, generalizing the Prime Ideal Principle for commutative rings due to T.Y. Lam and the author. Old and new "maximal implies prime" results are presented, with results touching on annihilator ideals, polynomial identity rings, the Artin-Rees property, Dedekind-finite rings, principal ideals generated by normal elements, strongly noetherian algebras, and just infinite algebras.
\end{abstract}

\section{INTRODUCTION}

A well-known phenomenon in ring theory is that, for certain properties $\mathcal{P}$, ideals of a ring that are maximal with respect to satisfying $\mathcal{P}$ are prime. This is probably most familiar in the setting of commutative algebra, as in [7. pp. 70-71, 84-85]. For instance, an ideal in any commutative ring $R$ is prime if it is maximal with respect to any of the following properties:

- being a proper subset of $R$,

- not being finitely generated,

- not being principal, or

- having empty intersection with a given multiplicative submonoid $S \subseteq R$.

The Prime Ideal Principle of [19] unifies the above results along with many other classical "maximal implies prime" results in commutative algebra. The basic idea behind this unification was to find certain properties of a family $\mathcal{F}$ of ideals in a fixed commutative ring that guarantee that an ideal maximal with respect to not lying in $\mathcal{F}$ is prime. The most notable such condition is that the family $\mathcal{F}$ be an Oka family; this means that, for any ideal $I \unlhd R$ and any element $a \in R$, the following implication holds:

$$
(I, a),(I: a) \in \mathcal{F} \Longrightarrow I \in \mathcal{F} \text {. }
$$

The Prime Ideal Principle then states that, for any Oka family $\mathcal{F}$ in a commutative ring $R$, an ideal $I \unlhd R$ maximal with respect to $I \notin \mathcal{F}$ is prime. All of the examples above were recovered from the Prime Ideal Principle in [19] by demonstrating that a relevant family of ideals is Oka.

A version of the Prime Ideal Principle for right ideals in noncommutative rings was established in [26. This was achieved by identifying an appropriate type of "prime right ideal"

Date: June 30, 2016.

2010 Mathematics Subject Classification. Primary: 16D25, 16N60; Secondary: 13A15, 16D20.

Key words and phrases. maximal implies prime, Prime Ideal Principle, Oka family of ideals.

This material is based upon work supported by the National Science Foundation under Grant No. DMS1407152. 
(these were called completely prime right ideals) and by finding a suitable definition of Oka families of right ideals. However, to date there is no version of the Prime Ideal Principle for two-sided ideals in noncommutative rings.

The purpose of this paper is to present such a theory for two-sided ideals in noncommutative rings, unifying many of the classical noncommutative "maximal implies prime" results and presenting some new ones. In Section 2 we briefly introduce Oka families of (two-sided) ideals in noncommutative rings and prove the corresponding Prime Ideal Principle: if $\mathcal{F}$ is an Oka family of ideals in a noncommutative $\operatorname{ring} R$ and $I$ is an ideal that is maximal with respect to $I \notin \mathcal{F}$, then $I$ is prime. We also introduce stronger conditions on families of ideals called $\left(P_{1}\right),\left(P_{2}\right)$, and $\left(P_{3}\right)$ that are noncommutative analogues of those defined in [19].

The heart of the paper lies in the remaining sections, which present many examples of families satisfying these different properties with corresponding applications of the Prime Ideal Principle. Section 3 gives examples of families satisfying the properties $\left(P_{1}\right),\left(P_{2}\right)$, and $\left(P_{3}\right)$. Section 4 considers families that satisfy the weaker $r$-Oka and Oka properties, many of which are constructed from various classes of (bi)modules; it concludes with a discussion of problems for future work.

Acknowledgments. I wish to thank George Bergman, T. Y. Lam, and Lance Small for helpful conversations, suggestions, and references to the literature. I also thank the referee for offering several clarifying remarks.

Conventions. Throughout this paper, all rings, modules, and ring homomorphisms are assumed to be unital. Let $R$ be a ring. We write $I \unlhd R$ to denote that $I$ is an ideal of $R$. If $X$ is a subset of $R$, we write $(X)$ for the ideal of $R$ generated by $X$; in case $X=\{x\}$ is a singleton we simply write $(x)=(\{x\})$. Furthermore, if $J \unlhd R$ and $a \in R$, then we denote $(I, J)=(I \cup J)=I+J$ and $(I, a)=(I \cup\{a\})=I+(\bar{a})$. The notation $M_{R}$ and ${ }_{R} M$ respectively indicate that $M$ is a right or left $R$-module.

\section{The Prime Ideal Principle}

The families of ideals defined below illustrate one possible way to generalize those ideal families defined in [19] from commutative rings to noncommutative rings. For two ideals $I, J \unlhd R$ we denote $J^{-1} I=\{x \in R: J x \subseteq I\}$ and $I J^{-1}=\{x \in R: x J \subseteq I\}$, both of which are clearly ideals in $R$.

Definition 2.1. Let $R$ be a ring and let $\mathcal{F}$ be a family of ideals of $R$ with $R \in \mathcal{F}$. We say that $\mathcal{F}$ is an Oka family of ideals if, for any $I \unlhd R$ and $a \in R$,

$$
(I, a),(a)^{-1} I, I(a)^{-1} \in \mathcal{F} \Longrightarrow I \in \mathcal{F} \text {. }
$$

We say that $\mathcal{F}$ is an $r$-Oka family of ideals if, for any $I \unlhd R$ and $a \in R$, the condition $(I, a),(a)^{-1} I \in \mathcal{F}$ implies that $I \in \mathcal{F}$. Furthermore, we define the following properties of the family $\mathcal{F}$ (recall the standing assumption that $R \in \mathcal{F}$ ), where $I, J \unlhd R$ are arbitrary ideals:

- Monoidal: $I, J \in \mathcal{F} \Longrightarrow I J \in \mathcal{F}$;

- Semifilter $: I \in \mathcal{F}$ and $J \supseteq I \Longrightarrow J \in \mathcal{F}$;

- $\left(P_{1}\right): \mathcal{F}$ is a monoidal semifilter;

- $\left(P_{2}\right): \mathcal{F}$ is monoidal, and for any $J \in \mathcal{F}, I^{2} \subseteq J \subseteq I \Longrightarrow I \in \mathcal{F}$;

- $\left(P_{3}\right)$ : For $A, B \in \mathcal{F}$ and $I \unlhd R, A B \subseteq I \subseteq A \cap B \Longrightarrow I \in \mathcal{F}$; 
- Strongly $r$-Oka: $I+J, J^{-1} I \in \mathcal{F} \Longrightarrow I \in \mathcal{F}$;

- Strongly Oka: $I+J, J^{-1} I, I J^{-1} \in \mathcal{F} \Longrightarrow I \in \mathcal{F}$.

The $r$-Oka and strongly $r$-Oka familes defined above have obvious left-handed analogues, which we refer to as (strongly) $\ell$-Oka families. An alternative term would have been "(strongly) right- and left-Oka" families. However, we have avoided this terminology since it would have suggested that an Oka family is the same as a family that is both right and left Oka. It seems likely that an Oka family need not be either $r$-Oka nor $\ell$-Oka in full generality (such as the families produced via Proposition 4.13), but we do not have an explicit example to verify this.

Each of the properties of ideal families listed above is easily seen to pass to arbitrary intersections of such families. Thus for each of these properties, the set of all ideal families in $R$ with that property forms a complete sublattice of the lattice of all families of ideals in $R$.

The following result outlines the logical dependence between the many types of families defined above.

Proposition 2.3. For a family $\mathcal{F}$ of ideals in a ring $R$, we have the following implications of the properties defined above:

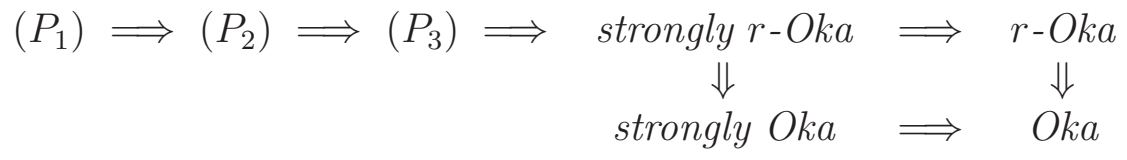

Proof. The horizontal implications are proved in the commutative case in [19, Thm. 2.7], and those arguments apply here with virtually no adjustment. The vertical implications are obvious.

We mention briefly that there are various equivalent characterizations of the $\left(P_{2}\right)$ and $\left(P_{3}\right)$ conditions given in [19, $\left.\S 2\right]$ and [20, $\left.\S 2\right]$. Though the statements and proofs are given for commutative rings, they once again transfer easily to the noncommutative case. Note that because $\left(P_{2}\right) \Rightarrow\left(P_{3}\right)$ and because any $\left(P_{3}\right)$ family is monoidal, a family satisfying any property $\left(P_{i}\right)$ is monoidal.

Before proving the Prime Ideal Principle in this setting, we establish a variant of the usual criterion for a ring to be prime. If $R$ is a prime ring and $a, b \in R \backslash\{0\}$ then one can conclude that both $a R b \neq 0$ and $b R a \neq 0$. There is an inherent symmetry in the definition of a prime ring in the sense that we can interchange the roles of $a$ and $b$. What if $R$ fails to be prime? We know offhand that there exist $a, b \in R \backslash\{0\}$ such that $a R b=0$. But if $R$ is not commutative, we cannot necessarily conclude that $b R a=0$. At first glance, the symmetry seems to disappear in the failure of a ring to be prime. The following lemma rectifies this situation.

Lemma 2.4. For any ring $R \neq 0$, the following are equivalent:

(1) $R$ is a prime ring

(2) For $a, b \in R \backslash\{0\}$, both $a R b \neq 0$ and $b R a \neq 0$

(3) For $a, b \in R \backslash\{0\}$, either $a R b \neq 0$ or $b R a \neq 0$.

In particular if $R$ is not a prime ring, then there exist $0 \neq a, b \in R$ such that $a R b=0=b R a$. 
Proof. The implications $(1) \Rightarrow(2) \Rightarrow(3)$ are immediate. To prove $(3) \Rightarrow(1)$ we will verify the contrapositive. Suppose that $R$ is not a prime ring; we will produce $a, b \in R \backslash\{0\}$ such that $a R b=b R a=0$. Because the ring is not prime, there exist $x, y \in R \backslash\{0\}$ such that $x R y=0$. If $y R x=0$ then we are finished. Otherwise there exists $r \in R$ such that yr $x \neq 0$. Setting $a=b=\operatorname{yr} x$ yields $a R b=b R a=0$.

We arrive at the following noncommutative version of the Prime Ideal Principle for twosided ideals in noncommutative rings. Given a family $\mathcal{F}$ of ideals in a ring $R$, its complement is $\mathcal{F}^{\prime}=\{I \unlhd R: I \notin \mathcal{F}\}$. We use $\operatorname{Max}\left(\mathcal{F}^{\prime}\right)$ to denote the set of ideals that are maximal in the complement of $\mathcal{F}$. Also, we let $\operatorname{Spec}(R)$ denote the set of prime ideals of $R$.

Theorem 2.5 (Prime Ideal Principle). For any Oka family $\mathcal{F}$ of ideals in a ring $R$, we have $\operatorname{Max}\left(\mathcal{F}^{\prime}\right) \subseteq \operatorname{Spec}(R)$.

Proof. Let $I \in \operatorname{Max}\left(\mathcal{F}^{\prime}\right)$, and assume for contradiction that $I$ is not prime. By Lemma 2.4 there exist $a, b \in R \backslash I$ such that $a R b, b R a \subseteq I$. Then $a \in(I, a) \backslash I$ and $b \in(a)^{-1} I \cap I(a)^{-1} \backslash I$. Maximality of $I$ implies that the ideals $(I, a),(a)^{-1} I$, and $I(a)^{-1}$ lie in $\mathcal{F}$. Because $\mathcal{F}$ is Oka, this means that $I \in \mathcal{F}$, a contradiction.

The Prime Ideal Principle supplement of [19] was a streamlined way to apply the Prime Ideal Principle in order to deduce that certain ideal-theoretic properties can be "tested" on the set of primes. We also have a noncommutative analogue of this result.

Theorem 2.6. Let $\mathcal{F}$ be an Oka family such that every nonempty chain of ideals in $\mathcal{F}^{\prime}$ (with respect to inclusion) has an upper bound in $\mathcal{F}^{\prime}$ (this holds, for example, if every ideal in $\mathcal{F}$ is finitely generated).

(1) Let $\mathcal{F}_{0}$ be a semifilter of ideals in $R$. If every prime ideal in $\mathcal{F}_{0}$ belongs to $\mathcal{F}$, then $\mathcal{F}_{0} \subseteq \mathcal{F}$.

(2) For an ideal $J \unlhd R$, if all prime ideals containing $J$ (resp. properly containing $J$ ) belong to $\mathcal{F}$, then all ideals containing $J$ (resp. properly containing $J$ ) belong to $\mathcal{F}$.

(3) If all prime ideals of $R$ belong to $\mathcal{F}$, then all ideals belong to $\mathcal{F}$.

Proof. Both (3) and (2) are special cases of (1). We prove (1) by a contrapositive argument. Let $\mathcal{F}_{0}$ be a semifilter such that $\mathcal{F}_{0} \nsubseteq \mathcal{F}$; we will construct a prime ideal in $\mathcal{F}_{0} \backslash \mathcal{F}$. Let $I_{0} \in \mathcal{F}_{0} \backslash \mathcal{F}=\mathcal{F}_{0} \cap \mathcal{F}^{\prime}$. The assumption on chains in $\mathcal{F}^{\prime}$ allows us to apply Zorn's lemma and find $I \in \operatorname{Max}\left(\mathcal{F}^{\prime}\right)$ such that $I_{0} \subseteq I$. The fact that $\mathcal{F}_{0}$ is a semifilter gives $I \in \mathcal{F}_{0}$. Because $I$ is maximal in the complement of $\mathcal{F}$, the Prime Ideal Principle 2.5 implies that $I$ is prime. Thus $I$ is a prime ideal in $\mathcal{F}_{0} \backslash \mathcal{F}$ as desired.

Remark 2.7. Another property of ideal families in commutative rings that was introduced in [19] was that of an Ako family; a family $\mathcal{F}$ in a commutative ring $R$ has the Ako property if $(I, a),(I, b) \in \mathcal{F}$ implies $(I, a b) \in \mathcal{F}$ for any $a, b \in R$. One can readily produce noncommutative versions of this property that satisfies the Prime Ideal Principle. For instance, $K$. Goodearl proposed the following noncommutative version of the Ako property: given $I \unlhd R$ and $a, b \in R$,

$$
(I, a),(I, b) \in \mathcal{F} \Longrightarrow(I, a r b) \in \mathcal{F} \text { for some } r \in R .
$$


Another possibility would be $(I, a),(I, b) \in \mathcal{F} \Longrightarrow I+(a)(b) \in \mathcal{F}$. One can readily verify that a family $\mathcal{F}$ satisfying either of these properties will also have $\operatorname{Max}\left(\mathcal{F}^{\prime}\right) \subseteq \operatorname{Spec}(R)$. Since the Ako property has seemed to be less useful than the Oka property already in the commutative setting, we do not focus our current efforts on this property.

Before proceeding to examples of Oka families, we conclude this section with a discussion of a prominent family of ideals that does not generally form an Oka family. One might wonder why a Prime Ideal Principle was given for one-sided ideals before it was established in the more classical setting of two-sided ideals. We will give a few examples to show that, in a sense, the case of two-sided ideals is more difficult to handle. Specifically, we recall the following classical result of Cohen [6, Thm. 2].

Cohen's Theorem. An ideal in a commutative ring that is maximal with respect to not being finitely generated is prime. Consequently, if every prime ideal of a commutative ring is finitely generated, then that ring is noetherian.

This result provided strong guidance for the creation of the Prime Ideal Principle in [19] and its noncommutative analogue in [26]. The behavior of the family of finitely generated directly motivated the definition of an Oka family of ideals in a commutative ring and for the definition of a right Oka family in a noncommutative ring.

In [26, Thm. 3.8] we provided a version of Cohen's result above for right ideals of a noncommutative ring. Specifically, after fixing an appropriate notion of an Oka family of right ideals, we proved that the family of finitely generated right ideals of any ring is an Oka family. As a consequence we showed that a right ideal of a ring that is maximal with respect to not being finitely generated is a completely prime right ideal. (Further Cohen-type results for right ideals in noncommutative rings were investigated in [27.)

A fundamental difference in the case of two-sided ideals of a noncommutative ring is that, if $R$ is not a commutative ring, then an ideal of $R$ that is maximal with respect to not being finitely generated need not be prime. This is illustrated in the following examples.

Let $A$ and $B$ be simple rings, and let $M$ be an $(A, B)$-bimodule. The triangular matrix ring

$$
R=T(A, M, B)=\left(\begin{array}{cc}
A & M \\
0 & B
\end{array}\right)
$$

will have exactly two prime ideals:

$$
\begin{aligned}
P_{1} & =\left(\begin{array}{cc}
A & M \\
0 & 0
\end{array}\right)=R\left(\begin{array}{ll}
1 & 0 \\
0 & 0
\end{array}\right) R \\
& =\left(\begin{array}{cc}
0 & M \\
0 & 0
\end{array}\right)+R\left(\begin{array}{ll}
1 & 0 \\
0 & 0
\end{array}\right)=\left(\begin{array}{cc}
0 & M \\
0 & 0
\end{array}\right)+\left(\begin{array}{ll}
1 & 0 \\
0 & 0
\end{array}\right) R, \\
P_{2} & =\left(\begin{array}{cc}
0 & M \\
0 & B
\end{array}\right)=R\left(\begin{array}{ll}
0 & 0 \\
0 & 1
\end{array}\right) R \\
& =\left(\begin{array}{cc}
0 & M \\
0 & 0
\end{array}\right)+R\left(\begin{array}{ll}
0 & 0 \\
0 & 1
\end{array}\right)=\left(\begin{array}{cc}
0 & M \\
0 & 0
\end{array}\right)+\left(\begin{array}{ll}
0 & 0 \\
0 & 1
\end{array}\right) R .
\end{aligned}
$$


The expressions above make it evident that $P_{1}$ and $P_{2}$ are always finitely generated as ideals, and that if $M$ is finitely generated as a left $A$-module (respectively, as a right $B$-module), then $P_{1}$ and $P_{2}$ will be finitely generated as left (respectively, right) ideals of $R$.

Example 2.8. Let $K$ be a field, let $M_{K}$ be an infinite-dimensional vector space viewed as a bimodule with the same left and right action, and consider the ring $R=T(K, M, K)$ (so $A=B=K$ above). Then $P_{1}$ and $P_{2}$ are finitely generated as ideals, but the ideal $I=\left(\begin{array}{ll}0 & V \\ 0 & 0\end{array}\right)$ is not finitely generated.

Example 2.9. Let $K / F$ be an infinite extension of fields. In the $(K, K)$-bimodule $M=$ $K \otimes_{F} K$, let $N$ be the sub-bimodule generated by $\{1 \otimes \alpha-\alpha \otimes 1: \alpha \in K\}$. This is a maximal sub-bimodule since $M / N \cong K$ as $(K, K)$-bimodules, and one can readily check that $M$ is not finitely generated (by choice of $K$ and $F$ ). In the ring $R=T(K, M, K$ ), the choice of $M$ and $N$ guarantees that the ideal $\left(\begin{array}{ll}0 & N \\ 0 & 0\end{array}\right)$ is maximal with respect to not being finitely generated as an ideal of $R$. But because $R / I \cong\left(\begin{array}{cc}K & K \\ 0 & K\end{array}\right)$, we see that $I$ is not even semiprime.

Example 2.10. We thank Lance Small for suggesting this example. Let $S$ be a simple ring that is not right noetherian (such as the endomorphism ring of a countably infinite dimensional vector space modulo its finite rank operators), let $Z=Z(S)$ be its central subfield, and set $R=T(Z, S, S)$. Then the prime ideals $P_{1}$ and $P_{2}$ of $R$ are both finitely generated as right ideals, but for any right ideal $J$ of $S$ that is not finitely generated, the corresponding ideal $\left(\begin{array}{ll}0 & J \\ 0 & 0\end{array}\right)$ of $R$ is not finitely generated.

Remark 2.11. Thanks to the examples given above, we see that for a general ring $R$ the family $\mathcal{F}_{\text {fg }}$ of finitely generated ideals of $R$ need not be an Oka family. For if $\mathcal{F}_{\text {fg }}$ were Oka and all prime ideals of $R$ were finitely generated, then by Theorem 2.6 all ideals of $R$ would be finitely generated, contrary to those examples. Similarly, the family of ideals that are finitely generated as right ideals is not an Oka family. These counterexamples sharply contrast the case of commutative rings [19, Proposition 3.16] and the case of Oka families of right ideals in noncommutative rings [26, Proposition 3.7].

The examples above naturally suggest the following question: do there exist simple rings $A$ and $B$ and an $(A, B)$-bimodule $M$ such that $M$ is finitely generated as both a left $A$ module and a right $B$-module, but such that $M$ has a sub-bimodule that is not finitely generated? For given such data, the ring $R=T(A, M, B)$ will have both prime ideals finitely generated as left ideals and as right ideals, but will have an ideal that is not finitely generated. Consequently, for this ring $R$ the family of ideals that are finitely generated both as left ideals and as right ideals would fail to be an Oka family. We suspect that such $(A, M, B)$ should exist, but we have yet to produce an explicit example.

\section{Some families satisfying the Properties $\left(P_{i}\right)$}

In this section we provide examples of families satisfying the conditions $\left(P_{1}\right),\left(P_{2}\right)$, and $\left(P_{3}\right)$. Comparing this section with the next, one sees that many well-known examples of the noncommutative "maximal implies prime" phenomenon come from such families. We begin with some of these classical examples.

Recall that a subset $S \subseteq R$ containing 1 is called an $m$-system if, for every $a, b \in S$, there exists $r \in R$ such that $a r b \in S$. It is well-known (and easy to show) that an ideal $I \unlhd R$ is 
prime if and only if $R \backslash I$ is an $m$-system. The notion of $m$-system generalizes the concept of a multiplicative subset of $R$, more common in commutative algebra. Our first application of the Prime Ideal Principle recovers a well-known generalization of the fact that an ideal in a commutative ring maximal with respect to being disjoint from some fixed multiplicative set is prime. The last part of this proposition is due to McCoy [22, Lem. 4].

Proposition 3.1. Let $S \subseteq R$ be an m-system. Then the family $\mathcal{F}=\{I \unlhd R: I \cap S \neq \varnothing\}$ is $\left(P_{1}\right)$. Hence an ideal maximal with respect to being disjoint from $S$ is prime, and every (nonzero) ideal of $R$ intersects $S$ if and only if every (nonzero) prime ideal intersects $S$.

Proof. Because $1 \in S$, we have $R \in \mathcal{F}$. It is clear that $\mathcal{F}$ is a semifilter. To see that $\mathcal{F}$ is monoidal, let $A, B \in \mathcal{F}$ and fix $a \in A \cap S$ and $b \in B \cap S$. Then for some $r \in R$, arb $\in \cap \cap A B$. Hence $A B \in \mathcal{F}$. The rest of the proposition follows from the Prime Ideal Principle 2.5.

Next we give an application of this result to the theory of algebras over a commutative ring $k$. Let $R$ be a $k$-algebra. We will say that an element $r \in R$ is integral over $k$ if, for all $r \in R$, there exists a monic polynomial $f(x) \in k[x]$ such that $f(r)=0$ in $R$. The algebra $R$ is integral over $k$ if every element of $R$ is integral over $k$; if $k$ is a field, notice that this is equivalent to $R$ being algebraic over $k$. The next result shows that, to check whether $R$ is integral over $k$, it suffices to check whether it is integral modulo each prime ideal. The fact is well-known; an instance of its application in the noncommutative setting is [24, Thm. 1].

Proposition 3.2. Let $R$ be an algebra over a commutative ring $k$. The family $\mathcal{F}=\{I \unlhd R$ : $R / I$ is integral over $k\}$ is $\left(P_{1}\right)$. In particular, $R$ is integral over $k$ if and only if $R / P$ is integral over $k$ for all (minimal) $P \in \operatorname{Spec}(R)$.

Proof. First, fix an element $a \in R$ and define the multiplicative set $S_{a}=\{f(a) \in R: f(x) \in$ $k[x]$ is monic $\}$. Then by Proposition 3.1 , the family $\mathcal{F}_{a}=\left\{I \unlhd R: I \cap S_{a} \neq \varnothing\right\}$ is $\left(P_{1}\right)$. Notice that $\mathcal{F}_{a}$ is precisely the family of ideals such that the image of $a$ in $R / I$ is integral over $k$. Now notice that the family $\mathcal{F}$ in question is given by $\mathcal{F}=\bigcap_{a \in R} \mathcal{F}$. Because $\mathcal{F}$ is an intersection of $\left(P_{1}\right)$ families, $\mathcal{F}$ itself is $\left(P_{1}\right)$. The last statement now follows from Theorem 2.6.

While on the subject of polynomials, we turn our attention to PI-algebras. We continue to assume that $k$ is a commutative ring. Let $k\left\langle x_{1}, x_{2}, \ldots\right\rangle$ denote the ring of polynomials over $k$ in noncommuting indeterminates $x_{i}$. Suppose that $R$ is a $k$-algebra and $f\left(x_{1}, \ldots, x_{n}\right) \in k\left\langle x_{i}\right\rangle$. Then we denote $f(R)=\left\{f\left(r_{1}, \ldots, r_{n}\right) \in R: r_{1}, \ldots, r_{n} \in R\right\}$. One says that $f$ is monic if one of its monomials of highest total degree has coefficient 1.

Lemma 3.3. If $f, g \in k\left\langle x_{1}, x_{2}, \ldots\right\rangle$ are monic, then $f g$ is monic.

Proof. Suppose that $f$ has degree $m$ and $g$ has degree $n$. Let

$$
\begin{aligned}
& f=a_{1} u_{1}+a_{2} u_{2}+\cdots+a_{r} u_{r}+(\text { lower degree terms }), \\
& g=b_{1} v_{1}+b_{2} v_{2}+\cdots+b_{s} v_{s}+\text { (lower degree terms), }
\end{aligned}
$$

where the $\left\{u_{i}\right\}$ are distinct words of length $m$ and the $\left\{v_{j}\right\}$ are distinct words of length $n$. We may assume that $a_{1}=b_{1}=1$ because $f$ and $g$ are monic. 
The highest degree terms of $f g$ are the products $a_{i} b_{j} u_{i} v_{j}$. Moreover, the monomials $u_{i} v_{j}$ are readily seen to be distinct. Hence $f g$ has $u_{1} v_{1}$ as one of its leading monomials, with coefficient $a_{1} b_{1}=1$, so that $f g$ is monic.

A $k$-algebra $R$ is a polynomial identity algebra (or PI algebra) over $k$ if $f(R)=0$ for some monic polynomial $f \in k\left\langle x_{1}, x_{2}, \ldots\right\rangle$ (see, for instance, [28, 6.1.2]). The last sentence of the following is a folk result; for example, it is used implicitly in the proof of [1, Thm. 2].

Proposition 3.4. Let $k$ be a commutative ring and let $S \subseteq k\left\langle x_{1}, x_{2}, \ldots\right\rangle$ be an $m$-system of polynomials in noncommuting indeterminates. For a $k$-algebra $R$, the family $\mathcal{F}=\{I \unlhd R$ : $R / I$ satisfies some $f \in S\}$ is $\left(P_{1}\right)$. Hence, an ideal maximal with respect to $R / I$ not being a $P I$-algebra over $k$ is prime.

Proof. Notice that $\mathcal{F}=\{I \unlhd R: f(R) \subseteq I$ for some $f \in S\}$. This makes it clear that $\mathcal{F}$ is a semifilter. To see that $\mathcal{F}$ is monoidal, suppose that $A, B \in \mathcal{F}$. Choose $f, g \in S$ and $h \in k\left\langle x_{1}, x_{2}, \ldots\right\rangle$ such that $f(R) \subseteq A$ and $g(R) \subseteq B$ as well as $f h g \in S$. Then $(f h g)(R) \subseteq f(R) h(R) g(R) \subseteq A B$ while $f h g \in S$, proving that $A B \in \mathcal{F}$.

For the last statement, we apply Theorem 2.5 to the family $\mathcal{F}$ taking $S$ to be the set of all monic polynomials in $k\left\langle x_{1}, x_{2}, \ldots\right\rangle$, which is multiplicatively closed by the lemma above.

We recall another familiar result from commutative algebra. For a module $M_{R} \neq 0$ over a commutative ring $R$, a point annihilator is an ideal $I \unlhd R$ of the form $I=\operatorname{ann}(m)$ for some $m \in M \backslash\{0\}$. Then a maximal point annihilator of $M$ is prime. But notice that, because $R$ is commutative, we have $\operatorname{ann}(m)=\operatorname{ann}(m R)$ for all $m \in M$. So a maximal point annihilator of $M$ is just an ideal maximal among the ideals of the form $\operatorname{ann}(N)$ where $0 \neq N_{R} \subseteq M$. From this perspective, the result generalizes to modules over noncommutative rings: for a module $M_{R} \neq 0$ over a ring $R$, an ideal maximal among the annihilators $\operatorname{ann}(N)$ where $0 \neq N_{R} \subseteq M$ is prime.

This well-known fact is widely attributed to I. N. Herstein; for instance, see [14, Thm. 6] (Lance Small has communicated to us that the first instance of this result is likely in [11].) We recover it via the Prime Ideal Principle below.

Proposition 3.5. Fix a module $M_{R} \neq 0$. Then the family $\mathcal{F}=\left\{J \unlhd R: N J=0\right.$ for $N_{R} \subseteq$ $M \Longrightarrow N=0\}$ is $\left(P_{1}\right)$. A maximal annihilator of a nonzero submodule of $M$ is prime.

Proof. It is straightforward to see that $\mathcal{F}$ is a semifilter. So suppose that $A, B \in \mathcal{F}$ and that $N_{R} \subseteq M$ such that $N(A B)=0$. Then $(N A) B=0 \Longrightarrow N A=0 \Longrightarrow N=0$.

To see that a maximal annihilator is prime, we only need to note that the maximal annihilators of nonzero submodules of $M$ are precisely the elements of $\operatorname{Max}\left(\mathcal{F}^{\prime}\right)$ and then apply the Prime Ideal Principle 2.5.

Applying this to the module $R_{R}$ gives the following result.

Proposition 3.6. In a nonzero ring $R$, the family $\mathcal{F}$ of ideals $I$ that are faithful as left $R$-modules is a $\left(P_{1}\right)$ family. If $R$ satisfies the ascending chain condition $(A C C)$ on ideals, then $R$ is a prime ring if and only if every nonzero prime ideal of $R$ is left faithful.

Proof. Applying Proposition 3.5 to $M_{R}=R_{R}$, we see that the family $\mathcal{F}$ there corresponds to the family of left-faithful ideals of $R$. Turning our attention to the final statement, notice 
that the "only if" part holds without the ACC assumption. The "if" part then follows from Theorem 2.6.

As a variation on the theme of maximal annihilator ideals, there are also maximal "middle annihilators." For ideals $X, Y \unlhd R$ we use the notation $\operatorname{ann}(X, Y)=\{r \in R: X r Y=0\}$, which is certainly an ideal of $R$. We call such an ideal a middle annihilator of $R$ if $X Y \neq 0$. This definition originated in unpublished work of Kaplansky (see [15, p. 782]), who proved the following "maximal implies prime" result.

Proposition 3.7. For any ring $R$, the family $\mathcal{F}=\{I \unlhd R$ : for all $X, Y \unlhd R, X I Y=0 \Longrightarrow$ $X Y=0\}$ is $\left(P_{1}\right)$. A maximal middle annihilator of $R$ is prime.

Proof. Certainly $\mathcal{F}$ is a semifilter. Suppose that $A, B \in \mathcal{F}$, and let $X, Y \unlhd R$ such that $X(A B) Y=0$. Then $(X A) B Y=0$ with $B \in \mathcal{F}$ implies that $X A Y=0$. But then $A \in \mathcal{F}$, so we must have $X Y=0$. Thus $A B \in \mathcal{F}$, proving that $\mathcal{F}$ is $\left(P_{1}\right)$. Because $\operatorname{Max}\left(\mathcal{F}^{\prime}\right)$ is equal to the set of maximal middle annihilators of $R$, the last statement follows from the Prime Ideal Principle 2.5.

Our next aim is to recover the well-known result that if the ideals in a ring $R$ satisfy ACC, then $R$ has finitely many minimal primes, along with a noncommutative generalization of a result of D. D. Anderson from [2] that any commutative ring in which the minimal primes are finitely generated has finitely many minimal primes. This makes use of the following.

Lemma 3.8. Let $I$ be a right ideal and $J$ be an ideal of a ring $R$. Suppose that these are generated as right ideals by $X \subseteq I$ and $Y \subseteq J$. Then the product $I J$ is generated as a right ideal by the set $\{x y: x \in X, y \in Y\}$. In particular, if $I$ and $J$ are finitely generated (resp. principal) as right ideals, then $I J$ is finitely generated (resp. principal) as a right ideal.

Proof. The second claim clearly follows from the first. Given $I$ and $J$ as above, the fact that $J$ is a (left) ideal gives the second equality below:

$$
\begin{aligned}
I J & =\left(\sum_{x \in X} x R\right) J \\
& =\sum_{x \in X} x J \\
& =\sum_{x \in X} x\left(\sum_{y \in Y} y R\right) \\
& =\sum_{(x, y) \in X \times Y} x y R .
\end{aligned}
$$

The following statement under hypothesis (1) is well known; for instance, see [10, Theorem 3.4]. The version of Anderson's theorem under hypothesis (2) was first suggested to us by T. Y. Lam.

Proposition 3.9. For any monoidal set $\mathcal{S}$ of ideals in $R$, the family $\mathcal{F}=\{I \unlhd R: I \supseteq$ $J$ for some $J \in \mathcal{S}\}$ is $\left(P_{1}\right)$. Suppose that one of the following two conditions holds:

(1) $R$ satisfies $A C C$ on ideals;

(2) The minimal prime ideals of $R$ are finitely generated as right ideals.

Then the zero ideal is a product of (minimal) prime ideals, and $R$ has finitely many minimal primes. 
Proof. It is clear that the family $\mathcal{F}$ is $\left(P_{1}\right)$. To prove the last statement, let $\mathcal{S}$ be the set of all finite products of minimal prime ideals of $R$ and let $\mathcal{F}$ be the $\left(P_{1}\right)$ family described in the Proposition. Notice that $\operatorname{Spec}(R) \subseteq \mathcal{F}$ because every prime contains a minimal prime. In the next paragraph, we will show that every nonempty chain in $\mathcal{F}^{\prime}$ has an upper bound in $\mathcal{F}^{\prime}$. Theorem 2.6 thus implies that $\mathcal{F}$ consists of all ideals. In particular $0 \in \mathcal{F}$, so that the zero ideal is a product of minimal primes, say $P_{1} \cdots P_{n}=0$. For any minimal prime $P \unlhd R$, we have $P \supseteq 0=P_{1} \cdots P_{n}$. So some $P_{i} \subseteq P$, giving $P=P_{i}$. Thus the minimal primes of $R$ are precisely $P_{1}, \ldots, P_{n}$.

It remains to verify that every nonempty chain in $\mathcal{F}^{\prime}$ has an upper bound. This is trivial in case (1) holds. If $R$ satisfies (2) then Lemma 3.8 implies that every ideal in $\mathcal{S}$ is finitely generated as a (right) ideal, whence the claim easily follows from Zorn's lemma.

Another notion that gives rise to prime ideals is the Artin-Rees property. An ideal $I \unlhd R$ is said to have the right Artin-Rees property if, for any right ideal $K_{R} \subseteq R$, there exists a positive integer $n$ such that $K \cap I^{n} \subseteq K I$. This property arises in the study of localization in noetherian rings (for example, see [10, Ch. 13] or [21, §4.2]). The ring $R$ is said to be a right Artin-Rees ring if every ideal of $R$ has the right Artin-Rees property.

(In commutative algebra, the Artin-Rees Lemma is a result about $I$-filtrations of finitely generated $R$-modules, where $R$ is commutative noetherian and $I \unlhd R$-see [7, Lem. 5.1]. In particular, the lemma implies that every ideal of a commutative noetherian ring satisfies the Artin-Rees property. Thus the family of ideals satisfying the right Artin-Rees property is mostly of interest in the noncommutative case.)

Parts of the following result were previously observed in [29, §3]. However, the "maximal implies prime" result seems to be new.

Proposition 3.10. The family of ideals with the right Artin-Rees property in a ring $R$ satisfies $\left(P_{2}\right)$. Thus an ideal of $R$ maximal with respect to not having the right Artin-Rees property is prime. In particular, if $R$ satisfies $A C C$ on ideals, then $R$ is a right Artin-Rees ring if and only if every prime ideal of $R$ has the right Artin-Rees property.

Proof. Let $\mathcal{F}$ be the family of ideals in $R$ with the right Artin-Rees property. Suppose that $J \in \mathcal{F}$ and $I \unlhd R$ with $I^{2} \subseteq J \subseteq I$. Fix a right ideal $K_{R} \subseteq R$ and an integer $n$ such that $K \cap J^{n} \subseteq K J$. Then $K \cap I^{2 n} \subseteq K \cap J^{n} \subseteq K J \subseteq K I$, which proves that $I \in \mathcal{F}$.

Next suppose that $A, B \in \mathcal{F}$. Fix a right ideal $K_{R} \subseteq R$, and choose integers $m$ and $n$ such that $K \cap A^{m} \subseteq K A$ and $(K A) \cap B^{n} \subseteq(K A) B$. Then for $N=\max \{m, n\}$, we have

$$
\begin{aligned}
K \cap(A B)^{N} & =K \cap(A B)^{m} \cap(A B)^{n} \\
& \subseteq\left(K \cap A^{m}\right) \cap B^{n} \\
& \subseteq(K A) \cap B^{n} \\
& \subseteq K A B .
\end{aligned}
$$

Thus $A B \in \mathcal{F}$ and $\mathcal{F}$ is monoidal. So $\mathcal{F}$ satisfies $\left(P_{2}\right)$. The last two statements follow from Theorems 2.5 and 2.6 .

The next family that we consider is the family of ideal direct summands of $R$; that is, the family of all ideals $I \unlhd R$ such that $R=I \oplus J$ for some ideal $J \unlhd R$. It is well-known that 
an ideal $I \unlhd R$ is such a direct summand if and only if $I=(e)$ for some central idempotent $e$ of $R$, in which case $J=(1-e$ ) (see, for instance, [18, Ex. 1.7]). In fact, we will work in the slightly more general setting of multiplicative sets of central idempotents.

Proposition 3.11. Let $R$ be a ring and let $S \subseteq R$ be a multiplicative set of central idempotents. Then the family $\mathcal{F}=\{(e) \unlhd R: e \in S\}$ satisfies $\left(P_{2}\right)$. In particular, the family of ideal direct summands of $R$ is $\left(P_{3}\right)$. An ideal maximal with respect to not being an ideal direct summand is maximal. A ring $R$ is a finite direct product of simple rings if and only if every ideal of $R$ is an ideal direct summand, if and only if all maximal ideals of $R$ are ideal direct summands.

Proof. To prove that $\mathcal{F}$ is monoidal, let $e, e^{\prime} \in S$ so that $(e)$ and $\left(e^{\prime}\right)$ are arbitrary ideals in $\mathcal{F}$. Then $e e^{\prime} \in S$, so we have $(e)\left(e^{\prime}\right)=\left(e e^{\prime}\right) \in \mathcal{F}$. Also, if $I \unlhd R$ with $(e)^{2} \subseteq I \subseteq(e)$, then $(e)^{2}=\left(e^{2}\right)=(e)$ implies that $I=(e) \in \mathcal{F}$. Thus $\mathcal{F}$ satisfies $\left(P_{2}\right)$.

Taking $S$ to be the multiplicative set of all central idempotents of $R$, the family $\mathcal{F}$ becomes the set of ideal direct summands of $R$. Now let $M \in \operatorname{Max}\left(\mathcal{F}^{\prime}\right)$. By the Prime Ideal Principle 2.5, $M$ is prime. In the factor $\operatorname{ring} R / M$, every ideal is generated by a central idempotent. We want to prove that $R / M$ is simple. Let $0 \neq I \unlhd R / M$ with $I=(e)$ for some central idempotent $e \in R / M$. Then $R / M=(e) \oplus(1-e)$, and $(e)(1-e)=(0)$. Because $R / M$ is prime and $(e) \neq 0$, we must have $(1-e)=0$. Thus $R / M=(e)=I$, proving that $R / M$ is simple.

The equivalence of the condition that all ideals are direct summands with the condition that all maximal ideals are summands follows from Theorem 2.6 along with the fact that every ideal in $\mathcal{F}$ is finitely generated. It remains to show that, if all ideals are direct summands, then $R$ is a finite direct product of simple rings (the converse being clear). If all ideals of $R$ are direct summands, then $R$ is a finitely generated semisimple module over the enveloping $\operatorname{ring} R^{e}=R \otimes_{\mathbb{Z}} R^{\mathrm{op}}$. By the classical theory of semisimple modules (see [17, §2]), this means that $R$ is a direct sum of simple $R^{e}$-submodules. But a simple $R^{e}$-submodule is a minimal ideal, and the desired equivalence easily follows.

Next we investigate the ideals $I \unlhd R$ such that $R / I$ is Dedekind finite. (Recall that a ring $R$ is Dedekind finite, alternatively called directly finite or von Neumann finite, if $a b=1$ implies $b a=1$ for any $a, b \in R$.)

Proposition 3.12. The family $\mathcal{F}=\{I \unlhd R: R / I$ is Dedekind finite $\}$ is $\left(P_{3}\right)$. An ideal maximal with respect to $R / I$ not being Dedekind finite is prime.

Proof. Let $A, B \in \mathcal{F}$ and $I \unlhd R$ with $A B \subseteq I \subseteq A \cap B$. We want to show that $I \in \mathcal{F}$. This is equivalent to showing that if $a, b \in R$ with $1-a b \in I$, then $1-b a \in I$. If $1-a b \in I \subseteq A \cap B$ then we must have $1-b a \in A \cap B$ because $A, B \in \mathcal{F}$. But then $1-2 b a+b a b a=(1-b a)^{2} \in$ $A B \subseteq I$. Also notice that $b a-b a b a=b(1-a b) a \in I$ because $1-a b \in I$. Thus

$$
1-b a=(1-2 b a+b a b a)+(b a-b a b a) \in I,
$$

proving that $I \in \mathcal{F}$. The last statement now follows from Theorem 2.5 .

Consideration of flat modules leads to the following example. Notice that the conclusion here that the family is $\left(P_{3}\right)$ is stronger than the one given in [19, (5.7)] when restricted to the commutative case. 
Proposition 3.13. Let $R$ be a ring and set $\mathcal{F}=\left\{I \unlhd R:(R / I)_{R}\right.$ is flat $\}$. Then $\mathcal{F}$ is a $\left(P_{3}\right)$ family. In particular, $\mathcal{F}$ is monoidal and an ideal $P$ maximal with respect to $(R / P)_{R}$ not being flat is a prime ideal.

Proof. Applying [16, (4.14)] to the exact sequence $0 \rightarrow I \rightarrow R \rightarrow R / I \rightarrow 0$, we see that $(R / I)_{R}$ is flat if and only if, for every left ideal ${ }_{R} L \subseteq R$ one has $I \cap L \subseteq I L$ (so that $I \cap L=I L)$. Thus

$$
\mathcal{F}=\left\{I \unlhd R: I \cap L \subseteq I L \text { for all }{ }_{R} L \subseteq R\right\} .
$$

Suppose that $I, A, B \unlhd R$ with $A, B \in \mathcal{F}$ and $A B \subseteq I \subseteq A \cap B$. Then $I \in \mathcal{F}$ because, for every ${ }_{R} L \subseteq R$, we have

$$
\begin{aligned}
I \cap L & \subseteq(A \cap B) \cap L \\
& =A \cap(B \cap L) \\
& =A \cap(B L) \\
& =(A B) L \\
& \subseteq I L .
\end{aligned}
$$

The last two statements follow from the the fact that $\left(P_{3}\right)$ families are monoidal and from the Prime Ideal Principle 2.5.

\section{OKA FAMILIES AND FAMILIES CONSTRUCTED FROM MODUle CATEGORIES}

In this final section, we provide examples of families that satisfy the $r$-Oka and Oka properties. Beginning with Proposition 4.7, these examples are produced from classes of modules that satisfy certain properties. Some of those examples in fact satisfy the strongest property $\left(P_{1}\right)$, but they are proved in this section as applications of the module-theoretic methods to be introduced.

Kaplansky showed [13, p. 486] that a commutative ring $R$ is a principal ideal ring (that is, every ideal of $R$ is principal) if and only if every prime ideal of $R$ is principal. Here we give a certain generalization of this result to noncommutative rings. (A different generalization for one-sided ideals was given in [27, Theorem 5.11].) We will say that an element $x \in R$ is normal if $x R=R x$, in which case we have $x R=(x)=R x$. This is equivalent to saying that both inclusions $x R \subseteq(x)$ and $R x \subseteq(x)$ are equalities. The set of normal elements is multiplicatively closed: if $x, y \in R$ are normal, then $x y R=x R y=R x y$.

Let us refine some of our notation. For a right ideal $I_{R} \subseteq R$ and an element $x \in R$, we define $x^{-1} I=\{y \in R: x y \in I\}$ which is a priori only a right ideal of $R$. However, if $I$ is a two-sided ideal and $x$ is normal, then one can easily verify that $x^{-1} I=(x)^{-1} I$, in which case $x^{-1} I$ is an ideal.

Proposition 4.1. If $S$ is a multiplicative set of normal elements in a ring $R$, then the set $\mathcal{F}=\{(s): s \in S\}$ is a strongly $r$-Oka family. Every ideal of a ring $R$ is generated by a single normal element if and only if every prime ideal in $R$ is generated by a normal element.

Proof. Let $I, J \unlhd R$ such that $(I, J), J^{-1} I \in \mathcal{F}$. Choose $x, y \in S$ such that $(I, J)=(x)$ and $J^{-1} I=(y)$. We claim that $I=(x y)$; because $x y \in S$ we will therefore have $I \in \mathcal{F}$. Noticing that $I \subseteq(I, J)=(x)=x R$, it is easy to verify that $I=x\left(x^{-1} I\right)$. But $x$ is normal, giving 
the first of the following equalities: $x^{-1} I=(x)^{-1} I=J^{-1} I=(y)$. Once more, normality of $x$ gives the final equality in $I=x\left(x^{-1} I\right)=x(y)=(x y)$.

For the last statement, we apply Theorem 2.6 to the family $\mathcal{F}$ taking $S$ to be the set of all normalizing elements.

In the special case of a right noetherian ring, for an ideal to be principally generated by a normal element, it suffices to test separately that it is principal as a left and a right ideal. The following may be a known folk result, but we became aware of it in a conversation with Konstantin Ardakov on Mathematics Stack Exchange 1

Lemma 4.2. Let $I$ be an ideal of a ring $R$ such that $I=a R=R b$ for some $a, b \in R$. Assume that one of the following hypotheses holds:

(1) $R$ is right noetherian;

(2) $R$ satisfies $A C C$ on principal right ideals and $a R$ is a projective right ideal (e.g., the right annihilator of a is zero).

Then $I=b R$ and $b$ is normal.

Proof. Fix $x, y \in R$ such that $a=x b$ and $b=a y$. Because $I$ is a left ideal, the function $\lambda(r)=x r$ restricts to a right $R$-module endomorphism of $I=a R$. This map is surjective because $a=x b=x a y$. In both cases (1) and (2), we will prove that $\lambda$ is an automorphism. Because $\lambda(b)=a$ implies that the submodule $b R \subseteq a R$ also has image equal to $\lambda(b R)=a R$, injectivity of $\lambda$ will in turn imply that $b R=a R$ as desired.

Under hypothesis (1), the right ideal $I$ is a noetherian (and therefore Hopfian) module, whence this surjection is an automorphism. Now suppose (2) holds, and assume toward a contradiction that $\lambda$ has kernel $0 \neq K_{1} \subseteq a R$. We can inductively define a chain of right ideals $K_{1} \subsetneq K_{2} \subsetneq \cdots$ by setting $K_{n} / K_{n-1}$ to be the (nonzero) kernel of the homomorphism $a R / K_{n-1} \cong a R \stackrel{\lambda}{\rightarrow} a R$. The third isomorphism theorem thus yields short exact sequences

$$
0 \rightarrow K_{n} \rightarrow a R \rightarrow a R \rightarrow 0
$$

for all $n$. Because $a R$ is projective, each of these sequences splits. Thus $K_{n}$, being a direct summand of the principal right ideal $a R$, is itself principal. Therefore the strictly ascending chain of principal right ideals $K_{n}$ contradicts condition (2).

Corollary 4.3. Let $R$ be a ring such that either:

(1) $R$ is right noetherian, or

(2) all principal right ideals of $R$ are projective (e.g., $R$ is a domain) and $R$ satisfies $A C C$ on principal right ideals.

Then any multiplicative submonoid of the family

$$
\mathcal{F}=\{I \unlhd R \mid I=a R=R b \text { for some } a, b \in R\}
$$

(including $\mathcal{F}$ itself) is an $r$-Oka family, and every ideal of $R$ is generated by a single normal element if and only if every prime ideal of $R$ is principal as both a left and right ideal.

\footnotetext{
${ }^{1}$ See http://math.stackexchange.com/q/627609
} 
Proof. By Lemma 3.8, the family $\mathcal{F}$ is closed under products. Next, Lemma 4.2 implies that every element of $\mathcal{F}$ is generated by a single normal element. The result now follows from Proposition 4.1.

We also mention the following immediate corollary of Lemma 4.2 ,

Corollary 4.4. If $R$ is a principal left and right ideal ring, then every ideal of $R$ is generated by a single normal element.

Now we turn our attention to invertible ideals. Let $R$ be a subring of a ring $Q$. We will say that an ideal $I \unlhd R$ is invertible with respect to $Q$ (or $Q$-invertible) if there exists an $(R, R)$-subbimodule $I^{*} \subseteq Q$ such that $I \cdot I^{*}=I^{*} \cdot I=R$. Certainly $I^{*}$ is unique. We claim that every invertible ideal in $R$ is finitely generated as a right ideal. To see this, let $I \unlhd R$ be invertible and choose $x_{i} \in I$ and $y_{i} \in I^{*}$ such that $\sum_{i=1}^{n} x_{i} y_{i}=1$. Then for $x \in I$ we have $x=\sum x_{i}\left(y_{i} x\right)$ where each $y_{i} x \in I^{*} I=R$. Thus $I=\sum x_{i} R$ is finitely generated as a right ideal. A symmetric argument proves that an invertible ideal is also finitely generated as a left ideal.

In the argument below, the reader is advised to take care not to confuse products $J^{*} I$ of $(R, R)$-bimodules inside of $Q$ with ideal quotients $J^{-1} I=\{x \in R: x J \subseteq I\}$.

Lemma 4.5. Let $R \subseteq Q$ be as above, and let $I, J \unlhd R$ where $I \subseteq J$ and $J$ is invertible. Then $I=J \cdot\left(J^{-1} I\right)$.

Proof. Notice that $I \subseteq J$ implies that $J^{*} I \subseteq J^{*} J=R$ is an ideal of $R$. Because $J\left(J^{*} I\right)=I$, it follows that $J^{*} I \subseteq J^{-1} I$. Left multiplying by $J$ gives $I=J\left(J^{*} I\right) \subseteq J\left(J^{-1} I\right) \subseteq I$, and the desired equality follows.

Proposition 4.6. Let $R$ be a subring of a ring $Q$. Then any monoidal family $\mathcal{F}$ of $Q$ invertible ideals of $R$ is a strongly $r$-Oka family. Hence an ideal maximal with respect to not being invertible is prime. Every nonzero ideal of $R$ is invertible if and only if every nonzero prime ideal of $R$ is invertible.

If every nonzero ideal of $R$ is $Q$-invertible, then the following hold:

(1) $R$ is a prime ring;

(2) If every nonzero ideal of $Q$ has nonzero intersection with $R$, then $Q$ is simple.

Proof. Let $I, J \unlhd R$ such that $(I, J), J^{-1} I \in \mathcal{F}$. Noting that $(I, J)^{-1} I=J^{-1} I$, we may apply Lemma 4.5 to find that $I=(I, J) \cdot\left(J^{-1} I\right) \in \mathcal{F}$. The second statement follows from Theorem 2.6 once we notice that the family of all $Q$-invertible ideals in $R$ is monoidal and every invertible ideal is finitely generated (as a right ideal).

Now assume that all nonzero ideals of $R$ are $Q$-invertible. Then (1) follows from the Prime Ideal Principle 2.5 because the zero ideal is maximal in the complement of the Oka family of all invertible ideals. Now assume that the hypothesis of (2) holds. To see that $Q$ is simple, let $K \unlhd Q$ be nonzero. Then $I=K \cap R$ is a nonzero ideal in $R$. Thus $I$ is invertible and consequently $1 \in R=I I^{*} \subseteq K I^{*} \subseteq K$. So $K=Q$, proving that $Q$ is simple.

Invertibility is typically studied in the context where $Q$ is a ring of quotients. For instance, let $R$ be a right Ore ring (that is, a ring in which the set of non-zero-divisors is a right Ore set $[16, \S 10 \mathrm{~B}]$ ) and let $Q$ be its classical right ring of quotients. (In this case, every right 
ideal of $Q$ has nonzero intersection with $R$, so the hypothesis of (B) above is satisfied.) If every nonzero prime ideal of $R$ is $Q$-invertible, the above implies that all nonzero ideals of $R$ are $Q$-invertible, that $R$ is prime, and that $Q$ is simple. When these conditions hold, $Q$ is (simple) artinian if and only if $R$ has finite right uniform dimension. Indeed, the simple ring $Q$ is equal to its own classical right ring of quotients. As it is prime and nonsingular, Goldie's theorem states that it is (right) artinian exactly when $Q$ has finite right uniform dimension. But $Q$ has finite right uniform dimension if and only if $R$ does (see [16, (10.35)]). (Of course, the ring of quotients $Q$ need not always be artinian if all ideals of $R$ are $Q$ invertible. For instance, consider that a ring which is a union of simple artinian subrings is again simple and equal to its own classical ring of quotients, but may fail to be artinian. Specifically we may take $R=Q$ to be the direct limit of the matrix rings $R_{n}=\mathbb{M}_{2^{n}}(k)$ for some division ring $k$, with $R_{n}$ considered as a subring of $R_{n+1}$ via the natural map $R_{n} \rightarrow R_{n} \otimes I_{2} \subseteq R_{n} \otimes_{k} \mathbb{M}_{2}(k) \cong R_{n+1}$. An explicit argument that $Q$ is not artinian is given in [17, p. 40].)

The remainder of the results in this section are based on families of ideals in a ring $R$ that are formed by considering those ideals $I$ such that the $(R, R)$-bimodule $R / I$ lies in a class $\mathcal{C}$ of bimodules, always assumed to contain the zero module, satisfying certain properties. We say that a class $\mathcal{C}$ of $(R, R)$-bimodules is closed under extensions if, for every short exact sequence of bimodules $0 \rightarrow L \rightarrow M \rightarrow N \rightarrow 0$, if $L, N \in \mathcal{C}$ then $N \in C$. (Taking $L=0$, note that this implies that if $M \cong N$ and $N \in \mathcal{C}$ then also $M \in \mathcal{C}$.) We say that $\mathcal{C}$ is closed under quotients if every homomorphic image of a bimodule in $\mathcal{C}$ is also in $\mathcal{C}$.

The first application of this kind of reasoning gives a method to construct examples of $\left(P_{1}\right)$ and $r$-Oka families. As one can tell from the hypotheses of $(2)$ and $(3)$ below, it applies especially well to right noetherian rings. In the following, for a module $M$ we use the term direct sum power of $M$ to mean a module that is a direct sum of copies of $M$, that is, a module of the form $M^{(X)}=\bigoplus_{X} M$ for some set $X$.

Proposition 4.7. Let $R$ be a ring and $\mathcal{C}$ a class of right $R$-modules containing the zero module, and denote $\mathcal{F}=\{I \unlhd R: R / I \in \mathcal{C}\}$.

(1) If $\mathcal{C}$ is closed under extensions, quotients, and arbitrary direct sum powers, then $\mathcal{F}$ satisfies $\left(P_{1}\right)$.

(2) Suppose that every ideal in $\mathcal{F}$ is finitely generated as a right ideal and that $\mathcal{C}$ is closed under extensions and quotients. Then $\mathcal{F}$ satisfies $\left(P_{1}\right)$.

(3) Suppose that every principal (equivalently, each finitely generated) ideal in $R$ is finitely generated as a right ideal and that $\mathcal{C}$ is closed under extensions and quotients. Then $\mathcal{F}$ is an $r$-Oka semifilter.

Proof. In each case, $\mathcal{F}$ is a semifilter because $\mathcal{C}$ is closed under quotients, and $R \in \mathcal{F}$ because $0 \in \mathcal{C}$. First suppose that $\mathcal{C}$ is as in (1). Let $I, J \in F$ and consider the short exact sequence of right $R$-modules

$$
0 \rightarrow \frac{I}{I J} \rightarrow \frac{R}{I J} \rightarrow \frac{R}{I} \rightarrow 0
$$

The right module $I / I J$ is annihilated by $J$, so for some sufficiently large set $X$ we have a surjection of $\bigoplus_{X} R / J$ (which is in $\mathcal{C}$ by closure under direct sum powers) onto $I / I J$ (which 
we now see to be in $\mathcal{C}$ by closure under quotients). Because $I / I J$ and $R / I$ belong to $\mathcal{C}$, which is closed under extensions, we have $R / I J \in \mathcal{C}$ and thus $I J \in \mathcal{F}$.

Next suppose that $\mathcal{F}$ and $\mathcal{C}$ are as in $(2)$, and fix $I, J \in \mathcal{F}$. The argument that $I J \in \mathcal{F}$ is identical to that for (1), taking into account the following modificiations. Because of the hypothesis on $\mathcal{F}, I \in \mathcal{F}$ implies that $I / I J$ is finitely generated as a right $R$-module. Thus the set $X$ above can be made finite, and because $\mathcal{C}$ is closed under extensions we obtain that $(R / J)^{X}$ lies in $\mathcal{C}$.

Now under the hypothesis of (3), let $I \unlhd R$ and $a \in R$ be such that $(I, a),(a)^{-1} I \in \mathcal{F}$. The assumption on $R$ implies that $(I, a) / I$ is a finitely generated right module in the short exact sequence

$$
0 \rightarrow \frac{(I, a)}{I} \rightarrow \frac{R}{I} \rightarrow \frac{R}{(a)^{-1} I} \rightarrow 0
$$

Further, the right annihilator of $(I, a) / I$ is equal to $(a)^{-1} I$. So we have a surjection of $R$ modules $\left(R /(a)^{-1} I\right)^{n} \rightarrow(I, a) / I$ for some positive integer $n$, and the argument can proceed as above to conclude that $I \in \mathcal{F}$. Hence $\mathcal{F}$ is an $r$-Oka family.

Example 4.8. Given an $m$-system $S$ in a $\operatorname{ring} R$, let $\mathcal{C}$ be the class of right $R$-modules $M$ such that $\operatorname{ann}(M) \cap S \neq \varnothing$. Then $\mathcal{C}$ is closed under extensions and quotients, because in any exact sequence $0 \rightarrow L \rightarrow M \rightarrow N \rightarrow 0$ of right $R$-modules, one has $\operatorname{ann}(N) \operatorname{ann}(L) \subseteq$ $\operatorname{ann}(M) \subseteq \operatorname{ann}(N)$. Also, $\operatorname{ann}\left(\bigoplus_{X} M\right)=\operatorname{ann}(M)$ for any indexing set $X$. So $\mathcal{C}$ satisfies the hypothesis of Proposition 4.7 (1) and satisfies $\left(P_{1}\right)$. Because ann $\left((R / I)_{R}\right)=I$ for any $I \unlhd R$, the family $\mathcal{F}=\{I \unlhd R: R / I \in \mathcal{C}\}$ is equal to the set of all ideals that have nonempty intersection with $S$. Thus we recover the same family that we considered in Proposition 3.1 .

Example 4.9. Let $R$ be a ring, and let $S \subseteq R$ be a multiplicative subset. Let $\mathcal{C}$ be the class of $S$-torsion modules: those modules in which every element is annihilated by an element of $S$. This class is easily seen to be closed under extensions, quotients, and arbitrary direct sums. It follows from Proposition 4.7(1) that the corresponding family

$$
\mathcal{F}=\{I \unlhd R: R / I \in \mathcal{C}\}=\left\{I \unlhd R: \text { for all } r \in R, r^{-1} I \cap S \neq \varnothing\right\}
$$

is $\left(P_{1}\right)$.

Two familiar facts in noncommutative ring theory state that (i) a ring is right artinian if and only if it is right noetherian and all of its prime factors are right artinian, and (ii) a left artinian ring is right artinian if and only if it is right noetherian. Here we improve these criteria for a ring to be right artinian by relaxing the right noetherian hypothesis in each case to the mere assumption that the two-sided ideals of the ring be finitely generated as right ideals.

Proposition 4.10. If every principal ideal of a ring $R$ is finitely generated as a right ideal, then the family $\mathcal{F}=\{I \unlhd R: R / I$ is right artinian $\}$ is $r$-Oka. A ring $R$ is right artinian if and only if $R / P$ is right artinian for all $P \in \operatorname{Spec}(R)$ and every ideal in $R$ is finitely generated as a right ideal. A left artinian ring is right artinian if and only if all of its ideals are finitely generated as right ideals.

Proof. The fact that $\mathcal{F}$ is $r$-Oka follows from Proposition 4.7)(3) applied to the class $\mathcal{C}$ of artinian right $R$-modules. 
If $R$ is right artinian, then certainly all of its prime factors are right artinian. Also $R$ is right noetherian, so its ideals are all finitely generated as right ideals. For the converse, suppose that all of the prime factors of $R$ are right artinian and that its ideals are finitely generated as right ideals. By the first part of this proposition, the family $\mathcal{F}$ defined above is strongly $r$-Oka. Because $\operatorname{Spec}(R) \subseteq \mathcal{F}$, the finiteness assumption on the ideals of $R$ allows us to conclude that all ideals lie in $\mathcal{F}$, according to Theorem 2.6. In particular we have $0 \in \mathcal{F}$, and $R$ is right artinian.

For the last statement, given a left artinian ring $R$ whose ideals are finitely generated as right ideals, we merely need to show that $R / P$ is right artinian for all $P \in \operatorname{Spec}(R)$. For each prime ideal $P \unlhd R$ the factor ring $R / P$ is left artinian and prime, hence semisimple and right artinian.

An even more general criterion for a left artinian ring to be right artinian is the result of D. V. Huynh [12, Theorem 1] that a left artinian ring is right artinian if and only if $J /\left(J^{2}+D\right)$ is right finitely generated where $J \unlhd R$ is the Jacobson radical of $R$ and $D \unlhd R$ is the maximal divisible torsion ideal.

A similar consideration gives a criterion for a ring to be right noetherian. We do not include the proof, which is identical to the proposition above. Notice that we do not have a corresponding result telling when a left noetherian ring is right noetherian due to the existence of prime left noetherian rings which are not right noetherian, such as a twisted polynomial extension $k[x ; \sigma]$ of a field $k$ by an endomorphism $\sigma: k \rightarrow k$ that is not surjective.

Proposition 4.11. If every principal ideal of a ring $R$ is finitely generated as a right ideal, then the family $\mathcal{F}=\{I \unlhd R: R / I$ is right noetherian $\}$ is r-Oka. A ring $R$ is right noetherian if and only if $R / P$ is right noetherian for all $P \in \operatorname{Spec}(R)$ and every ideal in $R$ is finitely generated as a right ideal.

The previous two results raise the question of whether the assumption on the ideals of $R$ can be further weakened. The following example shows that we cannot hope to do much

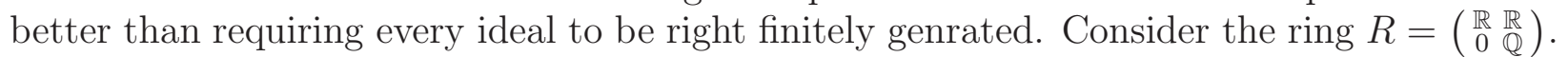
One can show (using, for example, [17, (1.17)]) that the only ideals in $R$ are

$$
0, I=\left(\begin{array}{cc}
0 & \mathbb{R} \\
0 & 0
\end{array}\right), P_{1}=\left(\begin{array}{cc}
\mathbb{R} & \mathbb{R} \\
0 & 0
\end{array}\right), P_{2}=\left(\begin{array}{ll}
0 & \mathbb{R} \\
0 & \mathbb{Q}
\end{array}\right) \text {, and } R \text {. }
$$

In particular we see that $R$ has finitely many ideals and thus satisfies both ACC and DCC on ideals. In addition, $R$ is not prime since $I^{2}=0$, and $\operatorname{Spec}(R)=\left\{P_{1}, P_{2}\right\}$ with $R / P_{1} \cong \mathbb{Q}$ and $R / P_{2} \cong \mathbb{R}$. Notice that $I$ is the smallest nonzero ideal of $R$ and that $R / I \cong \mathbb{R} \times \mathbb{Q}$ is semisimple, hence right artinian. Let $\mathcal{F}_{a}$ (resp. $\mathcal{F}_{n}$ ) denote the family of all ideals whose factor rings are right artinian (resp. right noetherian). Then we see that every nonzero ideal belongs to both $\mathcal{F}_{a}$ and $\mathcal{F}_{n}$, but the zero ideal is not prime. Then by the Prime Ideal Principle neither $\mathcal{F}_{a}$ nor $\mathcal{F}_{n}$ can be an Oka family. Additionally $R$ is left artinian but not right artinian or right noetherian (see [17, (1.22)]), even though it has finitely many ideals and all of its prime quotients are right noetherian.

Following Artin, Small, and Zhang [3], we say that an algebra $R$ over a commutative noetherian ring $C$ is strongly right noetherian if, for every commutative noetherian ring $D$ containing $C$ as a subring, the $D$-algebra $R \otimes_{C} D$ is right noetherian. (An example of Resco 
and Small [25] shows that a noetherian algebra over a field need not be strongly noetherian. On the other hand, Bell has shown [5] that this condition is automatically satisfied for a countably generated right noetherian algebra over an uncountable algebraically closed field.) These algebras play an important role in noncommutative algebraic geometry, especially in the theory of point modules [4].

Similar to the above examples, the strong right noetherian property provides another $r$-Oka family.

Proposition 4.12. Let $C$ be a commutative noetherian ring and let $R$ be a $C$-algebra. If every principal ideal of $R$ is finitely generated as a right ideal, then the semifilter

$$
\mathcal{F}=\{I \unlhd R: R / I \text { is a strongly right noetherian } C \text {-algebra }\}
$$

is $r$-Oka. If $R$ right noetherian, then it is strongly right noetherian over $C$ if and only if $R / P$ is strongly right noetherian for all (minimal) primes $P \in \operatorname{Spec}(R)$.

Proof. Let $\mathcal{C}$ be the class of all right $R$-modules $M$ such that, for any commutative noetherian overring $D \supseteq C$, the right $R \otimes_{C} D$-module $M \otimes_{C} D$ is noetherian. (For brevity, we write $M^{D}=M \otimes_{C} D$ below.) The statement will directly follow from Proposition 4.7 if we show that $\mathcal{C}$ is closed under extensions and quotients.

Fix a commutative noetherian overring $D \supseteq C$. Given a short exact sequence $0 \rightarrow L \rightarrow$ $M \rightarrow N \rightarrow 0$ of $R$-modules where $L, N \in \mathcal{C}$, right exactness of the tensor product gives an exact sequence $L^{D} \rightarrow M^{D} \rightarrow N^{D} \rightarrow 0$ of $R^{D}$-modules. Letting $K \subseteq M^{D}$ denote the kernel of $M^{D} \rightarrow N^{D}$, we obtain a short exact sequence of $R^{D}$ modules:

$$
0 \rightarrow K \rightarrow M^{D} \rightarrow N^{D} \rightarrow 0 .
$$

But $K$ Is a homomorphic image of the noetherian $R^{D}$-module $L^{D}$. So in the above, $K$ and $N^{D}$ are noetherian, implying that $M^{D}$ is noetherian. A similar argument using right exactness shows that $\mathcal{C}$ is also closed under quotient modules.

All of the examples of Oka families encountered up to this point actually satisfied stronger properties than the Oka condition. The next proposition will allow us to construct some Oka families without relying on any of the stronger conditions.

Proposition 4.13. Let $k$ be a commutative ring, and let $\mathcal{C}$ be a class of $k$-modules containing the zero module that is closed under quotients, extensions, and tensor products. Then for any $k$-algebra $R$, the family $\mathcal{F}=\{I \unlhd R: R / I \in \mathcal{C}\}$ is an Oka semifilter.

Proof. The family $\mathcal{F}$ is a semifilter because $\mathcal{C}$ is closed under quotients. To see that $\mathcal{F}$ is Oka, let $I \unlhd R$ and $a \in R$ be such that $(I, a),(a)^{-1} I, I(a)^{-1} \in \mathcal{F}$. Consider the following short exact sequence of $(R, R)$-bimodules:

$$
0 \rightarrow \frac{(I, a)}{I} \rightarrow \frac{R}{I} \rightarrow \frac{R}{(I, a)} \rightarrow 0 .
$$

We want to conclude that $R / I \in \mathcal{C}$. Because $R /(I, a) \in \mathcal{C}$ and $\mathcal{C}$ is closed under extensions, it suffices to show that $(I, a) / I \in \mathcal{C}$. Consider the $k$-bilinear map $R / I(a)^{-1} \times R /(a)^{-1} I \rightarrow$ $(I, a) / I$ given by

$$
\left(r+I(a)^{-1}, s+(a)^{-1} I\right) \mapsto \operatorname{ras}+I .
$$


This induces a $k$-module homomorphism $R / I(a)^{-1} \otimes_{k} R /(a)^{-1} I \rightarrow(I, a) / I$ that is evidently surjective. The fact that $\mathcal{C}$ is closed under tensor products and quotients then implies that $(I, a) / I \in \mathcal{C}$ as desired.

Part (2) of the following proposition gives a method of finding categories $\mathcal{C}$ satisfying the hypothesis of Proposition 4.13 .

Lemma 4.14. Let $k$ be a ring and let $\mathcal{C}$ be a class of $k$-modules containing the zero module.

(1) If $\mathcal{C}$ is closed under quotients and extensions, then it is closed under finite sums of modules in the following sense: if $N_{1}, \ldots, N_{m} \in \mathcal{C}$ are submodules of a single $k$-module $M$, then $\sum N_{i} \in \mathcal{C}$ also.

(2) Assume that $k$ is commutative. If $\mathcal{C}$ is closed under quotients and extensions and if $M, N \in \mathcal{C}$ with $N$ finitely generated, then $M \otimes_{k} N \in \mathcal{C}$. In particular, if every module in $\mathcal{C}$ is finitely generated, then $\mathcal{C}$ is closed under tensor products.

Proof. (1) It suffices to prove the statement for a sum of two submodules, for then an easy inductive argument extends the result to arbitrary finite sums. So suppose $N_{1}, N_{2}$ are submodules of the $k$-module $M$. Then we have the following short exact sequence:

$$
0 \rightarrow N_{1} \rightarrow N_{1}+N_{2} \rightarrow\left(N_{1}+N_{2}\right) / N_{1} \rightarrow 0
$$

But $\left(N_{1}+N_{2}\right) / N_{1} \cong N_{2} /\left(N_{1} \cap N_{2}\right) \in \mathcal{C}$ because $\mathcal{C}$ is closed under quotients. Thus the short exact sequence above gives $N_{1}+N_{2} \in \mathcal{C}$ as desired.

(2) Let $x_{1}, \ldots, x_{n} \in N$ form a generating set. Each of the submodules $M \otimes x_{i} \subseteq M \otimes N$ is a homomorphic image of $M$ (under the map $-\otimes x_{i}: M \rightarrow M \otimes x_{i}$ ) and thus lies in $\mathcal{C}$. But then $M \otimes N=\sum_{i=1}^{n} M \otimes x_{i} \in \mathcal{C}$.

Now we take a look at some specific examples.

Example 4.15. Let $R$ be an algebra over a commutative ring $k$. The family $\mathcal{F}$ of all ideals $I$ of $R$ such that $R / I$ is module-finite over $k$ is an Oka semifilter (using Proposition 4.13 applied to the class $\mathcal{C}$ of finitely generated $k$-modules). Thus, an ideal $I \unlhd R$ maximal with respect to having $R / I$ not module-finite over $k$ is prime.

The result above is related to the study of "just infinite" algebras. An algebra $R$ over a commutative ring $k$ is is said to be just infinite if $R$ is not finitely generated as a $k$-module, but every proper factor of $R$ is module-finite over $k$. A result attributed to Lance Small (see [28, Exercise 6.2.5], [23, Prop. 3.2], or [9, Lem. 2.1]) states that in case $k$ is a field, an infinite dimensional $k$-algebra has a just infinite factor ring (so that the family above has the Zorn property) which is prime.

The "maximal implies prime" statement above recovers part of Small's result. (For an alternative proof, see [8].) To recover the stronger result using the Zorn property, we require the following Artin-Tate-style lemma. We follow the proof strategy of [23, Lemma 3.1]. Recall that a $k$-algebra is affine if it is finitely generated as a $k$-algebra.

Lemma 4.16. Let $k$ be a commutative noetherian ring, and let $R$ be an affine $k$-algebra. If $M$ is a finitely generated right $R$-module with a submodule $L \subseteq M$ such that $M / L$ is finitely generated as a $k$-module, then $L$ is also finitely generated as an $R$-module. (In particular, if 
$I$ is a right ideal of $R$ such that $R / I$ finitely generated over $k$, then $I$ is finitely generated as a right ideal.)

Proof. Let $\left\{x_{1}, \ldots, x_{m}\right\}$ be $k$-algebra generating set of $R$ and let $\left\{g_{1}, \ldots, g_{n}\right\}$ be a set of $R$ module generators of $M$. Because $M / L$ is finitely generated over $k$, there exist $v_{1}, \ldots, v_{t} \in M$ such that, for $V=\sum k v_{i}$, we have $M=L+V$.

Consequently there exist elements $p_{\alpha} \in L$ and $q_{\beta \gamma} \in L$ such that each $g_{\alpha} \in p_{\alpha}+V$ and each $v_{\beta} x_{\gamma} \in q_{\beta \gamma}+V$. Let $L_{0} \subseteq L$ be the $R$-submodule generated by the finite set of the $p_{\alpha}$ and $q_{\beta \gamma}$. It is straightforward to see that $L_{0}+V$ is an $R$-submodule of $M$. By construction, each $g_{\alpha} \in L_{0}+V$, so $M=L_{0}+V$.

Finally, if $l \in L$ then $l=l_{0}+v$ for $l_{0} \in L_{0} \subseteq L$ and $v \in V$. But also $v=l-l_{0} \in L$. It follows that $L=L_{0}+(L \cap V)$. Because $k$ is noetherian and $V$ is finitely generated over $k$, the $k$-submodule $L \cap V$ is also finitely generated over $k$. Thus $L=L_{0}+(L \cap V)$ is finitely generated as an $R$-module.

Theorem 4.17. Let $R$ be an affine algebra over a commutative noetherian ring $k$. The family

$$
\mathcal{F}=\{I \unlhd R: R / I \text { is finitely generated as a } k \text {-module }\}
$$

satisfies $\left(P_{1}\right)$. Thus any just infinite $k$-algebra is prime. Furthermore, an algebra $R$ that is not finitely generated as a $k$-module is just infinite over $k$ if and only if $R / P$ is module-finite over $k$ for all nonzero $P \in \operatorname{Spec}(R)$.

Proof. The previous lemma implies that every ideal in $\mathcal{F}$ is finitely generated as a right ideal. Applying Proposition 4.7(2) to the class $\mathcal{C}$ of right $R$-modules that are finitely generated over $k$, we conclude that $\mathcal{F}$ is a $\left(P_{1}\right)$ family. The final two claims respectively follow from the Prime Ideal Principle 2.5 and Theorem 2.6.

We present two more examples of Oka families derived from Proposition 4.13, In both examples, $k$ denotes a commutative ring.

Example 4.18. The class of noetherian $k$-modules is closed under quotients and extensions, and consists of finitely generated modules. Then as above, the family of all $I \unlhd R$ with $R / I$ noetherian over $k$ is an Oka semifilter.

Example 4.19. The class of $k$-modules of finite (composition) length, which is the same as the category of finitely generated artinian $k$-modules, is closed under quotients and extensions and consists of finitely generated modules. So the family of $I \unlhd R$ such that $R / I$ is artinian over $k$ is an Oka semifilter.

We conclude with some thoughts on future directions in the study of the "maximal implies prime" phenomenon for two-sided ideals in noncommutative rings. When comparing the theories of Oka families for commutative rings [19] and for right ideals in noncommuative rings [26] with the theory developed above, we notice one striking difference. In both of the former two settings, it was shown that Oka families in a ring $R$ are in bijective correspondence with classes of cyclic $R$-modules that are closed under extensions (see [19, §4] and [26, §4]). This allows one to take any module-theoretic property that is preserved by extensions and produce a corresponding Oka family, a method which led to a number of novel examples of "maximal implies prime" results in both cases. 
By contrast, we have not found any (bi)module-theoretic characterization of the Oka families of Definition 2.1 leading to a correspondence between Oka families in a ring $R$ and certain classes of (bi)modules. While Propositions 4.7 and 4.13 provide sufficient conditions for a class of bimodules to give rise to an Oka family, both of these methods seem severely limited by the fact that they can only produce Oka semifilters of ideals. (Note that each of those results involved a short exact sequence of $(R, R)$-bimodules the form

$$
0 \rightarrow \frac{(I, a)}{I} \rightarrow \frac{R}{I} \rightarrow \frac{R}{(I, a)}
$$

where $I$ is an ideal and $a$ is an element of the ring $R$. In each case, the hypotheses are imposed with the intent of finding a description of $(I, a) / I$ in terms of the bimodules $R / I(a)^{-1}$ and $R /(a)^{-1} I$. The key noncommutative difficulty seems to be the lack of such a description in full generality.)

Thus, in conclusion, we offer two general problems to stimulate further work in this direction.

(1) Is it possible to characterize Oka families of ideals in a noncommutative ring $R$ in terms of suitable classes of $(R, R)$-bimodules (perhaps using a more appropriate definition than the one given in Definition 2.1)?

(2) In lieu of such a characterization, is there a related method that yields more examples of Oka families that are not semifilters and that do not satisfy conditions $\left(P_{1}\right)-\left(P_{3}\right)$ ?

Even partial progress on these problems seems likely to lead to new "maximal implies prime" results for ideals in noncommutative rings.

\section{REFERENCES}

1. S. A. Amitsur and Lance W. Small, Algebras over infinite fields, revisited, Israel J. Math. 96 (1996), no. part A, 23-25. MR 1432724 (97k:16025)

2. D. D. Anderson, A note on minimal prime ideals, Proc. Amer. Math. Soc. 122 (1994), no. 1, 13-14. MR 1191864 (94k:13001)

3. M. Artin, L. W. Small, and J. J. Zhang, Generic flatness for strongly Noetherian algebras, J. Algebra 221 (1999), no. 2, 579-610. MR 1728399 (2001a:16006)

4. M. Artin and J. J. Zhang, Abstract Hilbert schemes, Algebr. Represent. Theory 4 (2001), no. 4, 305-394. MR 1863391 (2002h:16046)

5. Jason P. Bell, Noetherian algebras over algebraically closed fields, J. Algebra 310 (2007), no. 1, 148-155. MR 2307786 (2008f:16050)

6. I. S. Cohen, Commutative rings with restricted minimum condition, Duke Math. J. 17 (1950), 27-42. MR $0033276(11,413 \mathrm{~g})$

7. David Eisenbud, Commutative Algebra: with a View Toward Algebraic Geometry, Graduate Texts in Mathematics, vol. 150, Springer-Verlag, New York, 1995. MR 1322960 (97a:13001)

8. John Farina and Cayley Pendergrass-Rice, A few properties of just infinite algebras, Comm. Algebra $\mathbf{3 5}$ (2007), no. 5, 1703-1707. MR 2317639 (2008b:16030)

9. Daniel R. Farkas and Lance W. Small, Algebras which are nearly finite dimensional and their identities, Israel J. Math. 127 (2002), 245-251. MR 1900701 (2003c:16033)

10. K. R. Goodearl and R. B. Warfield, Jr., An introduction to noncommutative Noetherian rings, second ed., London Mathematical Society Student Texts, vol. 61, Cambridge University Press, Cambridge, 2004. MR 2080008 (2005b:16001)

11. I. N. Herstein, Due risultati classici sugli anelli, Univ. e Politec. Torino Rend. Sem. Mat. 21 (1961/1962), 99-102. MR 0148689 (26 \#6196) 
12. Dinh Van Huynh, A note on rings with chain conditions, Acta Math. Hungar. 51 (1988), no. 1-2, 65-70. MR 934584 (89e:16024)

13. Irving Kaplansky, Elementary divisors and modules, Trans. Amer. Math. Soc. 66 (1949), 464-491. MR 0031470 (11,155b)

14. _ Commutative Rings, revised ed., The University of Chicago Press, Chicago, Ill.-London, 1974. MR 0345945 (49 \#10674)

15. Günter Krause, Middle annihilators in Noetherian rings, Comm. Algebra 8 (1980), no. 8, 781-791. MR 566421 (81h:16025)

16. T. Y. Lam, Lectures on Modules and Rings, Graduate Texts in Mathematics, vol. 189, Springer-Verlag, New York, 1999. MR 1653294 (99i:16001)

17. _ A First Course in Noncommutative Rings, second ed., Graduate Texts in Mathematics, vol. 131, Springer-Verlag, New York, 2001. MR 1838439 (2002c:16001)

18. __ Exercises in Classical Ring Theory, second ed., Problem Books in Mathematics, Springer-Verlag, New York, 2003. MR 2003255 (2004g:16001)

19. T. Y. Lam and Manuel L. Reyes, A Prime Ideal Principle in commutative algebra, J. Algebra 319 (2008), no. 7, 3006-3027. MR 2397420

20. _ Oka and Ako ideal families in commutative rings, Rings, modules and representations, Contemp. Math., vol. 480, Amer. Math. Soc., Providence, RI, 2009, pp. 263-288. MR 2508156 (2010j:13008)

21. J. C. McConnell and J. C. Robson, Noncommutative Noetherian Rings, revised ed., Graduate Studies in Mathematics, vol. 30, American Mathematical Society, Providence, RI, 2001, With the cooperation of L. W. Small. MR 1811901 (2001i:16039)

22. Neal H. McCoy, Prime ideals in general rings, Amer. J. Math. 71 (1949), 823-833. MR 0032590 (11,311c)

23. D. S. Passman and W. V. Temple, Representations of the Gupta-Sidki group, Proc. Amer. Math. Soc. 124 (1996), no. 5, 1403-1410. MR 1307556 (96g:20009)

24. C. Procesi, The Burnside problem, J. Algebra 4 (1966), 421-425. MR 0212081 (35 \#2956)

25. Richard Resco and L. W. Small, Affine Noetherian algebras and extensions of the base field, Bull. London Math. Soc. 25 (1993), no. 6, 549-552. MR 1245080 (94m:16029)

26. Manuel L. Reyes, A one-sided prime ideal principle for noncommutative rings, J. Algebra Appl. 9 (2010), no. 6, 877-919. MR 2748128 (2012a:16007)

27. - Noncommutative generalizations of theorems of Cohen and Kaplansky, Algebr. Represent. Theory 15 (2012), no. 5, 933-975. MR 2969284

28. Louis H. Rowen, Ring Theory, Student Edition, Academic Press Inc., Boston, MA, 1991. MR 1095047 (94e:16001)

29. Boris Širola, On noncommutative Noetherian schemes, J. Algebra 282 (2004), no. 2, 667-698. MR 2101080 (2006b:16050)

Department of Mathematics, Bowdoin College, 8600 College Station, Brunswick, Me 04011-8486, USA

E-mail address: reyes@bowdoin.edu

URL: http://www.bowdoin.edu/〜reyes/ 